\title{
Diosgenin protects against alveolar bone loss in ovariectomized rats via regulating long non-coding RNAs
}

\author{
ZHIGUO ZHANG ${ }^{1}$, YANJING CHEN ${ }^{1}$, LIHUA XIANG ${ }^{1}$, ZHEN WANG $^{1}$, \\ GARY GUISHAN XIAO ${ }^{2,3}$ and DAHONG JU ${ }^{4}$
}

\begin{abstract}
${ }^{1}$ Institute of Basic Theory, China Academy of Chinese Medical Sciences, Beijing 100700; ${ }^{2}$ School of Pharmaceutical Science, Dalian University of Technology, Dalian, Liaoning 116024, P.R. China; ${ }^{3}$ Functional Genomics and Proteomics Laboratory,

Osteoporosis Research Center, Creighton University Medical Center, Omaha, NE 68131 USA;

${ }^{4}$ Experimental Research Center, China Academy of Chinese Medical Sciences, Beijing 100700, P.R. China
\end{abstract}

Received January 23, 2018; Accepted August 23, 2018

DOI: $10.3892 /$ etm.2018.6681

\begin{abstract}
The present study assessed the changes in long non-coding (lnc)RNA and mRNA expression profiles when diosgenin (DIO) exerted a potential osteoprotective effect on the alveolar bone of ovariectomized (OVX) rats. Female Wistar rats underwent a sham operation (SHAM group) or ovariectomy. OVX rats were treated using vehicle (OVX group), DIO (DIO group) or estradiol valerate (EV group) for 12 weeks. After treatment, the biomarkers of bone turnover in plasma and the microstructure of alveolar bone were assessed. lncRNA microarrays were applied to assess lncRNA and mRNA expression profiles in alveolar bone in the OVX and DIO group rats. Subsequently, the differentially expressed mRNAs associated with the comprehensive bone metabolism pathway in Ingenuity Pathway Analysis (IPA) were identified and regarded as key mRNAs. Based on some of the key mRNAs and all the differentially expressed lncRNAs, a coexpression network was established and this network was further analyzed to identify the top 6 lncRNAs with the highest closeness scores (pivotal lncRNAs). Finally, 6 modules showing interactions between pivotal lncRNAs and key mRNAs were constructed. All of the pivotal lncRNAs and key mRNAs were validated with reverse transcription-quantitative polymerase chain reaction. The present findings demonstrated that DIO suppressed the loss of alveolar bone in OVX rats, and the changes to the expression of some lncRNAs or mRNAs
\end{abstract}

Correspondence to: Professor Dahong Ju, Experimental Research Center, China Academy of Chinese Medical Sciences, 16 Nanxiaojie, Dongzhimennei, Beijing 100700, P.R. China

E-mail: judahong@126.com

Professor Gary Guishan Xiao, School of Pharmaceutical Science, Dalian University of Technology, 2 Linggong Road, Ganjingzi, Dalian, Liaoning 116024, P.R. China

E-mail: gxiao@dlut.edu.cn

Key words: diosgenin, alveolar bone, osteoprotective effect, long noncoding RNAs, coexpression network occurred in the alveolar bone of the rats in the DIO group. Twenty-four key mRNAs were identified during pathway analysis. Furthermore, 8/24 key mRNAs (Ctnnb1, Smad4, Tcf2, Sp7, Illb, Illrl, Tnf and Tnfrsfla) were used to establish a coexpression network, which included 1,656 nodes and 5,341 edges. During network analysis, 6 pivotal lncRNAs (XR_008346, MRuc007iji, MRAK157089, MRAK076413, MRAK143591 and AB036696) were obtained, and 6 modules illustrating pivotal lncRNA-key mRNA interactions were identified. These results revealed that the anti-osteoporotic effect of DIO on alveolar bone may be associated with the promotion of a bone formation process through increasing the signaling of the Wnt and BMPs pathways and the inhibition of the bone resorption process through decreasing stimulators of osteoclastogenesis. To conclude, several pivotal lncRNAs may serve important roles in these processes via regulating some key mRNAs in the bone metabolism pathway.

\section{Introduction}

Postmenopausal women suffering from osteoporosis have a higher risk of tooth and periodontal disease, which might be related to alveolar bone loss $(1,2)$. The metabolic mechanism of alveolar bone is partly different from that of other bones, and its biological metabolic cycle is very short (3). Diosgenin (DIO), an aglycone of the steroid saponin, is widely recognized as a phytoestrogen $(4,5)$, but the estrogen-like effect of DIO is milder than real estrogen. A study indicated that DIO, at doses of $20-200 \mathrm{mg} / \mathrm{kg}$, did not affect the uterine wet weight, epithelium height, volume densities of endometrium, endometrial epithelia, number of endometrial glands, or histological appearance of vaginal epithelia in rats (6). In addition, DIO shows adverse effects such as stimulatory action on growth of mammary gland in ovariectomized (OVX) mouse (7). As for bone metabolism, previous studies demonstrated that the application of DIO decreases bone loss in the peripheral skeleton in animal models induced by ovariectomy $(8,9)$, but the effects of DIO on alveolar bone still remain unclear. We therefore conducted a study to assess the influence of DIO on alveolar bone in rats that had undergone ovariectomy. 
Long noncoding RNAs (lncRNAs), defined as RNAs $>200$ nucleotides and lacking an open reading frame (ORF), were identified from cDNA in $1991(10,11)$. Like microRNAs (miRNAs), lncRNAs are also considered to play important roles in normal cellular physiological processes, with tissueand cell-specific expression patterns $(12,13)$. The limited studies on lncRNAs showed that they might play a vital role in the occurrence and progression of skeletal diseases $(14,15)$ including osteoporosis $(16,17)$. In studies on animal models, some specific lncRNAs could be potential biomarkers for the diagnosis of osteoporosis in the jaw of OVX mice have been reported (18).

In view of the importance of lncRNAs in bone remodeling, we questioned if IncRNAs play a substantial role in the action of DIO on the loss of alveolar bone. Therefore, we conducted this study to assess the modulatory action of DIO on lncRNA and mRNA expression profiles in alveolar bone in an OVX rat model.

\section{Materials and methods}

Animal grouping and treatments. There have been studies that used female rats aged 6 months that have received an ovariectomy as a model of osteoporosis in postmenopausal women (19,20). Forty-eight female Wistar rats aged 6 months, with an average weight of $300 \pm 20.0 \mathrm{~g}$, were obtained from the Experimental Animal Center of the Academy of Military Medical Sciences. All the experiments on animals gained approval of the Institutional Ethics Committee of the Institute of Basic Theory, China Academy of Chinese Medical Sciences. The rats were sham-operated (SHAM, $n=12$ ) or bilaterally OVX (n=36) (21). We randomly divided the OVX rats into the following three groups: OVX group (OVX, $n=12)$, DIO group (DIO, $n=12)$, and estradiol valerate (EV) group ( $\mathrm{EV}, n=12)$. We treated the rats in the EV group with EV daily via oral gavage at a dosage of $0.1 \mathrm{mg} / \mathrm{kg}$ body weight/day. In addition, we treated the rats in the DIO group with DIO via oral gavage at a dosage of $100 \mathrm{mg} / \mathrm{kg}$ body weight/day. At the same time, we treated the rats in the SHAM and OVX groups with an equivalent volume of distilled water via oral gavage. During the experiments, we fed all the rats with standard chow. We started all of the treatments 1 week following OVX surgery, and the course continued for 12 weeks. During the 12-week treatment period, no animal died.

Preparation of specimens. The average body weight of all rats was $365.84 \pm 26.85 \mathrm{~g}$ in the end of treatment. We anesthetized the animals with xylazine at $12 \mathrm{mg} / \mathrm{kg}$ and ketamine at $80 \mathrm{mg} / \mathrm{kg}$ via intraperitoneal injection and then euthanized them by exsanguination the day after the last treatment. Under anesthesia, the abdominal aorta of rat was punctured to collect 8-10 $\mathrm{ml}$ of blood specimens into heparinized tubes. After exsanguinations, we performed cardiac palpation on precordium of rats for five min to confirm cardiac arrest. If the rats showed cardiac arrest, respiratory arrest and mydriasis simultaneously, we confirmed the death of rat. Subsequently, the blood specimens were separated by centrifugation at $3,000 \times \mathrm{g}$ at a temperature of $4^{\circ} \mathrm{C}$ for $10 \mathrm{~min}$ and then aliquoted and preserved at $-80^{\circ} \mathrm{C}$ before use. We dissected the right mandibles and then stored them at $-20^{\circ} \mathrm{C}$ for determination of microstructure and bone mineral density (BMD) with the help of micro-computerized tomography (micro-CT). Afterwards, we used them for histological examination. We dissected the left mandibles and reserved the bone tissue between the incisor and molars and afterwards stored it at $-80^{\circ} \mathrm{C}$ for reverse transcription-quantitative polymerase chain reaction (RT-qPCR) tests and microarray assay.

Biomarkers of bone turnover. Enzyme-linked immunosorbent assays (ELISAs; Beijing Sunbio Biotech Co., Ltd., Beijing, China) were conducted to assess plasma concentrations of bone resorption and bone formation biomarkers, such as tartrate-resistant acid phosphatase (TRAP) and alkaline phosphatase (ALP), in controlled, standardized and duplicated experiments. An ELISA reader (BioTek Instruments, Inc., Winooski, VT, USA) was used to read absorption at $450 \mathrm{~nm}$.

micro-CT assessments. We scanned the right mandible of each rat with a high-resolution micro-CT system (Skyscan 1172 micro-CT system; Bruker Corporation, Ettlingen, Germany) without sample preparation. We applied the micro-CT system in accordance with a method described previously (22). We scanned each of the samples at a high resolution $(6.8 \mu \mathrm{m})$. We applied a $0.5 \mathrm{~mm}$ thick aluminum filter to remove noise from the generated gray scale images. A global threshold (lower grey threshold: 52, upper grey threshold: 255) was used for measurement of the trabecular bone.

We captured the images of the mandible at a current of $100 \mu \mathrm{A}$ and a voltage of $100 \mathrm{keV}$ to reconstruct a cubic region starting $1.5 \mathrm{~mm}$ below the bottom of the first molar crown, which served as the 'volume of interest' (VOI). We applied the standard Skyscan software package for trabecular bone morphological measurements within the VOI. In addition, we applied 3-D analyses for assessment of the trabecular bone volume fraction (BV/TV), the BMD, the trabecular separation (Tb.Sp), the trabecular thickness (Tb.Th), the trabecular number (Tb.N), the degree of anisotropy (DA) and the structural model index (SMI) for the same VOI (23).

Histological examination. We fixed the right mandibles in formalin at a concentration of $10 \%$, decalcified mandible in EDTA at a concentration of $14 \%$, dehydrated them, and then embedded them in paraffin. The cutting of sections was performed with a standard microtome, and then the cut sections were affixed to glass slides and stained sections using hematoxylin and eosin.

Analysis of lncRNA microarray data. We prepared alveolar bone from six rats from the DIO and OVX groups. The Rat LncRNA Array (Arraystar Inc., Rockville, MD, USA) was used for profiling the lncRNAs and protein-coding mRNAs in one experiment. In the microarray, there were approximately 9300 lncRNAs from the UCSC all_mRNA records, NCBI RefSeq and orthologs of rat lncRNAs databases and 15200 protein-coding mRNAs from the NCBI RefSeq database that were evaluated at the same time.

We performed the IncRNA microarray assay at KangChen Bio-tech (Shanghai, China). In brief, we isolated total RNA with TRIzol reagent (Invitrogen; Thermo Fisher Scientific, Inc., Waltham, MA, USA) in accordance with the instructions. 
We amplified one milligram of the total RNA from each group and transcribed itinto fluorescent complementary RNA (cRNA). Then, we hybridized the labeled cRNAs onto a $4 \times 44 \mathrm{~K}$ Rat LncRNA Array. After washing the slides, we scanned the arrays using an Agilent G2505B Microarray Scanner. The analysis of the acquired array images was performed using Agilent Feature Extraction software (v.10.7.3.1; Agilent Technologies, Inc., Santa Clara, CA, USA). Finally, we performed quantile normalization using Expander v5.1 software and processed the data subsequently with the GeneSpring GX v.11.5.1 software package (Agilent Technologies, Inc.). Threshold values of a fold change $\geq 2$ and P-value $<0.05$ were used for screening of differentially expressed lncRNAs and mRNAs.

Ingenuity pathway analysis (IPA). We imported the differentially expressed mRNAs from array analyses into the Ingenuity Pathway Analysis Tool. The IPA Tool identifies global functions, canonical pathways and biological networks of a particular dataset on the basis of the Ingenuity Pathways Knowledge Base (IPKB). Among the canonical pathways of IPKB, the pathway 'Role of Osteoblasts, Osteoclasts and Chondrocytes in Rheumatoid Arthritis (ROOCRA)' is unique and covers almost all important molecules and signaling associated with functions of osteoblasts and osteoclasts, such as differentiation, mineralization, degradation, development, and apoptosis. Considering the importance of the ROOCRA pathway in bone metabolism, we further searched the differentially expressed mRNAs allocated into the ROOCRA pathway, and these mRNAs were considered key mRNAs.

Build of mRNA-lncRNA coexpression network. We selected all of the differentially expressed lncRNAs and some of the key mRNAs to build the coexpression network according to the method described previously $(24,25)$. The building of the network involved the following procedures: i) preprocessing data- we used the median value of one mRNA with different transcripts to represent the gene expression, without exceptional treatment for lncRNA expression values; ii) screening data-we removed the subsets of data in accordance with the lists of the different kinds of lncRNA and selected mRNA; iii) then, we calculated the Pearson's correlation coefficient and applied the P-value for calculations between lncRNAs and key mRNAs; and iv) screening on the basis of Pearson's correlation coefficients-we considered values over 0.98 meaningful and used them to draw the mRNA-lncRNA coexpression network using Cytoscape (v.2.8.3; http://www. cytoscape.org) (26).

Analysis of mRNA-lncRNA coexpression network. Closeness centrality is concerned with the shortest distance of a node to all the other nodes in a network. Closeness centrality of a certain node is the combined shortest distance from all other nodes. Closeness centrality represents the potent influence of a node on the network (27). The closeness scores of nodes in the coexpression network were calculated using the CytoHubba plug-in (28) in Cytoscape. We selected six lncRNAs with the highest closeness scores and considered them pivotal lncRNAs. Furthermore, six modules showing interaction between pivotal lncRNAs and key mRNAs were built.
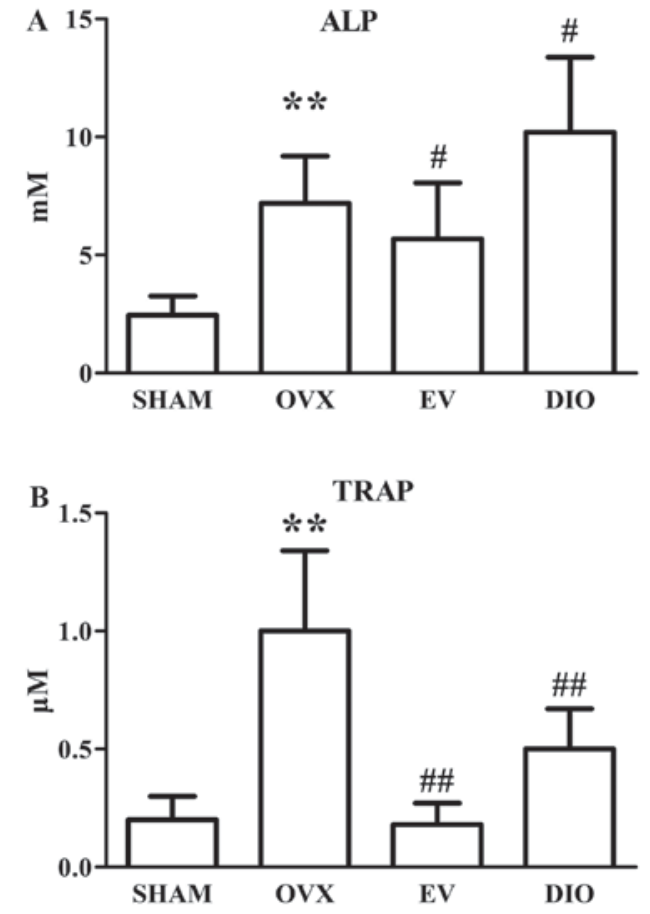

Figure 1. Influence of DIO on levels of ALP and TRAP in plasma after a 12-week treatment. (A) ALP, (B) TRAP. ${ }^{* *} \mathrm{P}<0.01$ vs. SHAM group; ${ }^{*} \mathrm{P}<0.05$ vs. OVX group. SHAM, sham-operation; DIO, diosgenin; OVX, ovariectomy; EV, estradiol valerate; ALP, alkaline phosphatase; TRAP, tartrate-resistant acid phosphatase.

Validation of differentially expressed mRNAs and lncRNAs using $R T$-qPCR. We performed RT-qPCR analyses for lncRNAs and mRNAs with SYBR RT-qPCR kits (Takara Biotechnology Co., Ltd., Dalian, China) and an ABI 7500 system (Applied Biosystems; Thermo Fisher Scientific, Inc.) in accordance with the instructions. We used an internal control, Gapdh, to normalize the comparative expression level of lncRNAs or mRNAs with the $2^{-\Delta \Delta C t}$ cycle threshold technique.

Statistical analysis. We expressed the data as the mean \pm standard deviation. In addition, we used the Student-Newman-Keuls post hoc tests and one-way ANOVA for determination of significant differences between results using SPSS v.19.0 statistical software package (IBM Corp., Armonk, NY, USA). $\mathrm{P}<0.05$ was considered to indicate a statistically significant difference.

\section{Results}

Influence of DIO on bone turnover biomarkers. The plasma concentrations of ALP and TRAP in different rats after a 12-week treatment are shown in Fig. 1A, B. After 12-week treatment, the ALP and TRAP levels in OVX rats $(7.20 \pm 2.01 \mathrm{mM}$, $1.01 \pm 0.34 \mu \mathrm{M}$, respectively) were significantly higher than those in SHAM rats $(2.45 \pm 0.80 \mathrm{mM}, 0.22 \pm 0.11 \mu \mathrm{M}$, respectively, $\mathrm{P}<0.01)$. Moreover, the plasma ALP and TRAP levels in $\mathrm{EV}$ group rats $(5.68 \pm 2.38 \mathrm{mM}, 0.18 \pm 0.09 \mu \mathrm{M}$, respectively) were significantly lower than those in OVX group rats $(\mathrm{P}<0.05$ and $\mathrm{P}<0.01)$. Nevertheless, DIO increased the level of ALP $(10.20 \pm 3.18 \mathrm{mM})$ and decreased the level of TRAP 

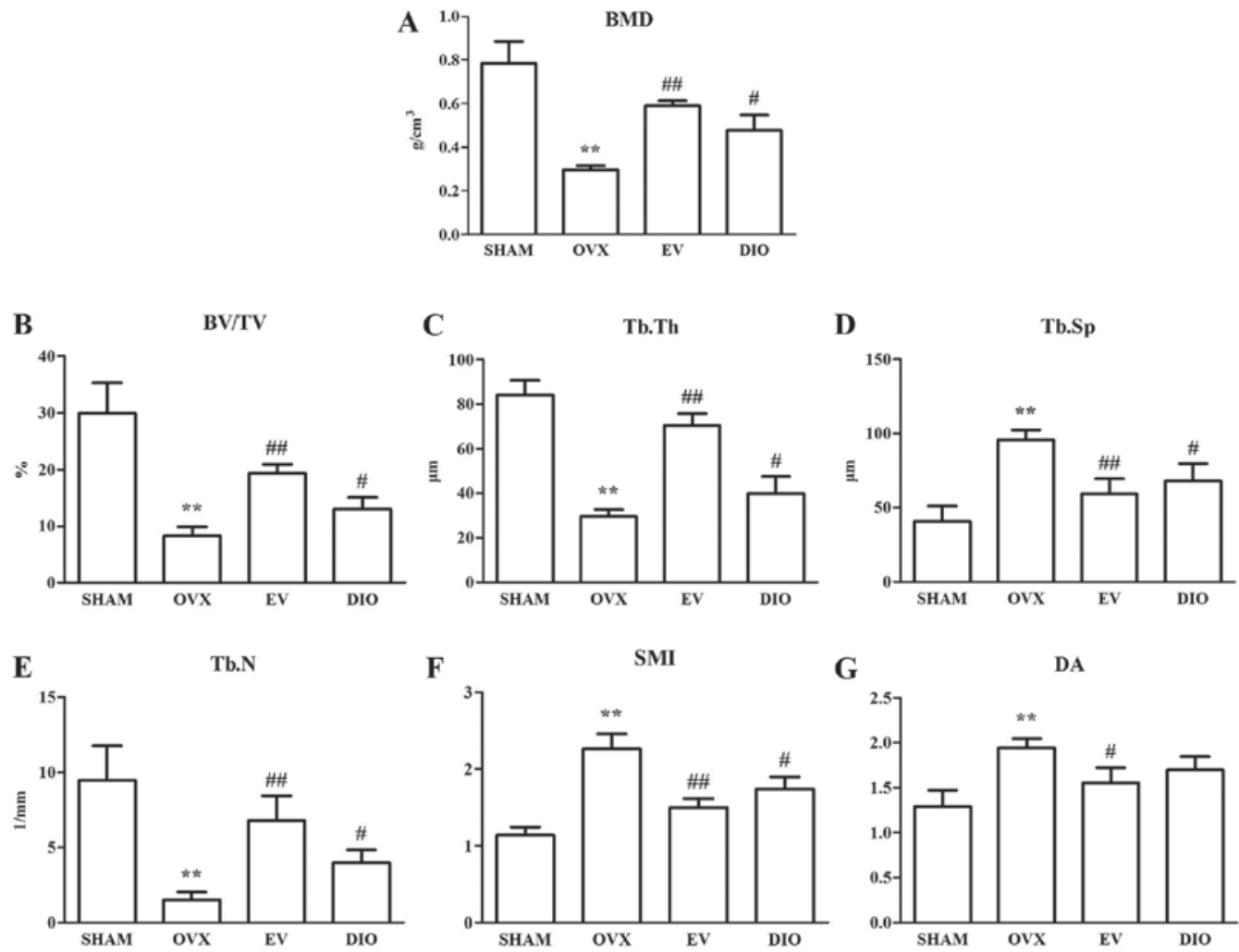

Figure 2. Influence of DIO on BMD and trabecular bone microarchitecture after 12-week treatment. (A) BMD, (B) BV/TV, (C) Tb.Th, (D) Tb.Sp, (E) Tb.N (F) SMI and (G) DA. ${ }^{* *} \mathrm{P}<0.01$ vs. SHAM group; ${ }^{\# /} \mathrm{P}<0.01$ vs. OVX group; ${ }^{*} \mathrm{P}<0.05$ vs. OVX group. SHAM, sham-operation; OVX, ovariectomy; EV, estradiol valerate; DIO, diosgenin. SHAM, sham-operation; OVX, ovariectomy; EV, estradiol valerate; DIO, diosgenin; BMD, bone mineral density; BV/TV, trabecular bone volume fraction; Tb.Sp, trabecular separation; Tb.Th, trabecular thickness; Tb.N, trabecular number; DA, degree of anisotropy; SMI, structural model index

$(0.50 \pm 0.17 \mu \mathrm{M})$ in rats compared to those in OVX group rats $(\mathrm{P}<0.05$ and $\mathrm{P}<0.01)$.

Influence of DIO on trabecular bone microstructure and BMD. The evaluation of trabecular bone microstructure of the four groups was conducted with micro-CT. The results suggested that ovariectomy obviously reduced the BMD, Tb.N, Tb.Th and BV/TV (BMD: 0.296 $\pm 0.019 \mathrm{~g} / \mathrm{cm}^{3}, \mathrm{~Tb} . \mathrm{N}: 1.530 \pm 0.510 \mathrm{~mm}^{-1}$, Tb.Th: $29.636 \pm 2.830 \mu \mathrm{m}, \mathrm{BV} / \mathrm{TV}: 8.308 \pm 1.5840 \%, \mathrm{P}<0.01)$ and increased the SMI, Tb.Sp, and DA (SMI: 2.267 \pm 0.189 , Tb.Sp: $95.443 \pm 7.045 \mu \mathrm{m}$, DA: $1.943 \pm 0.101$, respectively, $\mathrm{P}<0.01)$ in comparison with the SHAM group (BMD: 0.786 $\pm 0.099 \mathrm{~g} / \mathrm{cm}^{3}$, BV/TV: $29.952 \pm 5.371 \%$, Tb.Th: $84.001 \pm 6.540 \mu \mathrm{m}, \mathrm{Tb} . \mathrm{Sp}$ : 40.646 $\pm 10.107 \mu \mathrm{m}$, Tb.N: $9.480 \pm 2.280 \mathrm{~mm}^{-1}$, SMI: $1.137 \pm 0.108$, DA: $1.290 \pm 0.180)$ based on the morphometric parameters of alveolar bone. The OVX-induced changes were significantly inhibited after treatment with EV (BMD: $0.589 \pm 0.025 \mathrm{~g} / \mathrm{cm}^{3}, \mathrm{BV} / \mathrm{TV}: 19.321 \pm 1.567 \%$, Tb.Th: $70.454 \pm 5.180 \mu \mathrm{m}$, Tb.Sp: $59.053 \pm 10.360 \mu \mathrm{m}, \mathrm{Tb} . \mathrm{N}$ : $6.810 \pm 1.610 \mathrm{~mm}^{-1}$, SMI: $\left.1.498 \pm 0.120, \mathrm{DA}: 1.555 \pm 0.167\right)$ or DIO (BMD: $0.477 \pm 0.071 \mathrm{~g} / \mathrm{cm}^{3}, \mathrm{BV} / \mathrm{TV}: 12.964 \pm 2.072 \%$, Tb.Th: $39.893 \pm 7.550 \mu \mathrm{m}$, Tb.Sp: $68.063 \pm 11.601 \mu \mathrm{m}, \mathrm{Tb} . \mathrm{N}$ : $3.990 \pm 0.840 \mathrm{~mm}^{-1}$, SMI: $1.741 \pm 0.157$, DA: $\left.1.700 \pm 0.145\right)$ (Fig. 2A-G). Furthermore, impairment of the trabecula caused by ovariectomy was relieved after treatment with EV or DIO (Fig. 3A-D)

Influence of DIO on histomorphology of alveolar bone. A histological examination was conducted to observe the alveolar bone region of the first molar. The typical histological sections of SHAM or OVX rats treated with vehicle, EV or DIO are shown in Fig. 4. Sections from rats in the SHAM group demonstrated thickened alveolar bone and a small and scant medullary cavity (Fig. 4A) relative to those from the OVX group. OVX significantly decreased the volume of alveolar bone and increased the size of the medullary cavity (Fig. 4B). Alveolar bone volume was significantly increased after 12 weeks of treatment with EV or DIO (Fig. 4C and D), and the effect of EV was greater than that of DIO. We mutually confirmed the results of histological observations and micro-CT.

Influence of DIO on lncRNA and mRNA expression profile. Microarray data analysis confirmed that there were 2409 differentially expressed lncRNAs and 3145 differentially expressed mRNAs in alveolar bone from the DIO group in comparison to those from the OVX group rats. Among the 2409 lncRNAs, 1311 were upregulated and 1098 were down-regulated. Among the 3145 mRNAs, 1468 were shown upregulated and 1677 mRNAs were shown down-regulated.

Influence of DIO on ROOCRA pathway. A total of 24 differentially expressed mRNAs (key mRNAs) in rat alveolar bone between the DIO group and the OVX group were allocated into the ROOCRA pathway (Table I). Figs. 5 and 6 are schematic diagrams illustrating the role of these key mRNAs in osteoblasts and osteoclasts based on the ROOCRA pathway, respectively. 
A

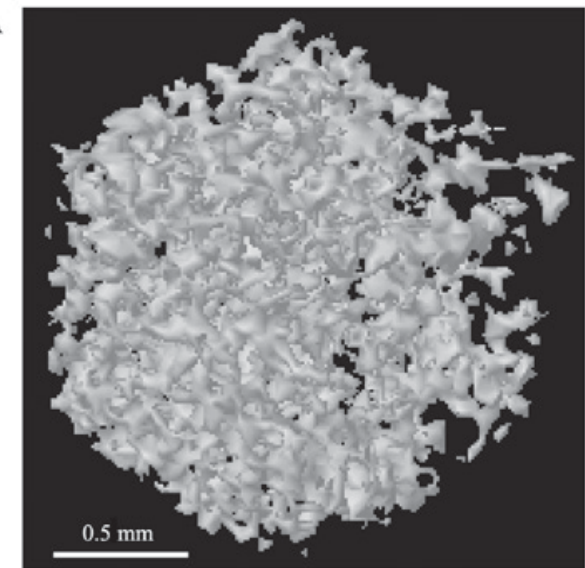

C

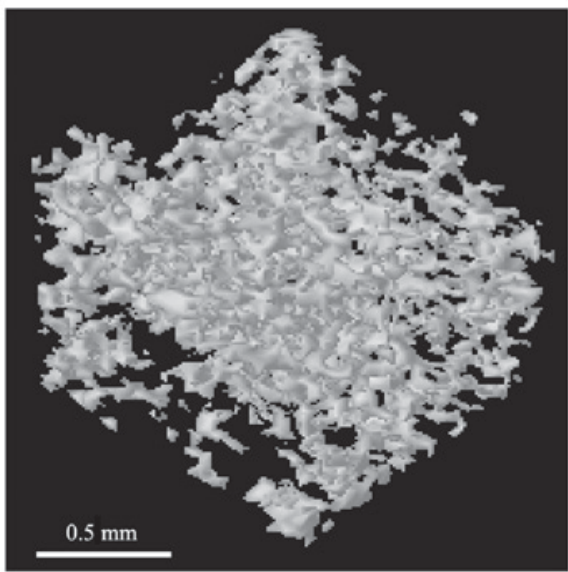

B

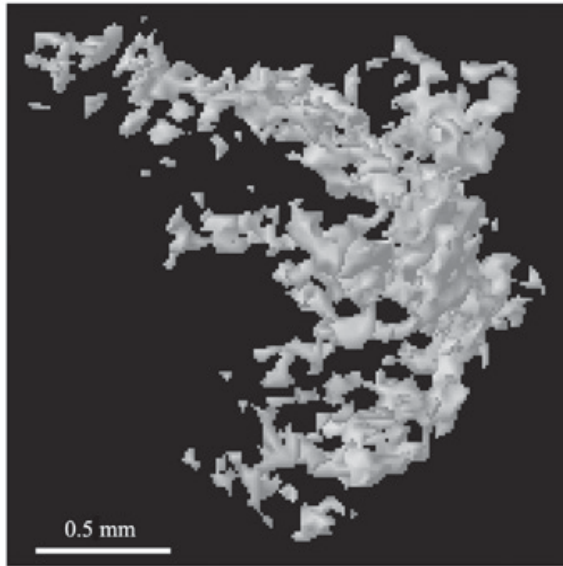

D

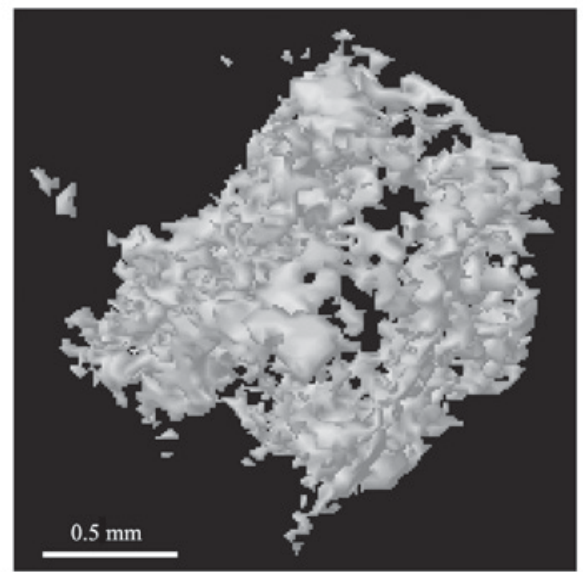

Figure 3. Typical samples from each group: 3-D structure of alveolar bone below the bottom of the first molar. (A) SHAM, (B) OVX, (C) EV and (D) DIO. SHAM, sham-operation; DIO, diosgenin; OVX, ovariectomy; EV, estradiol valerate.

A

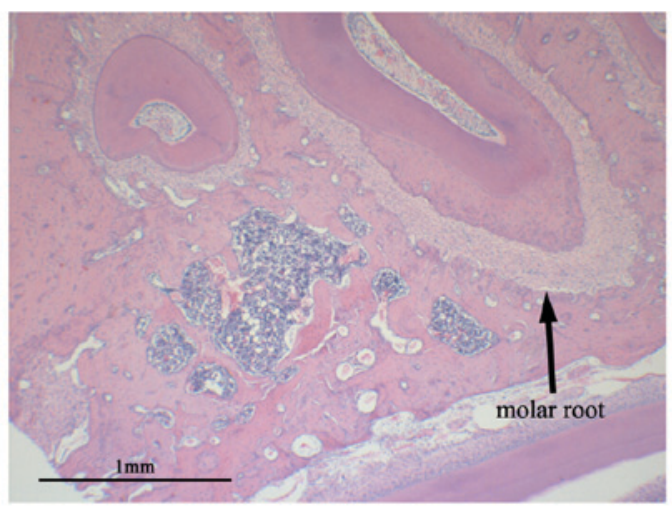

C

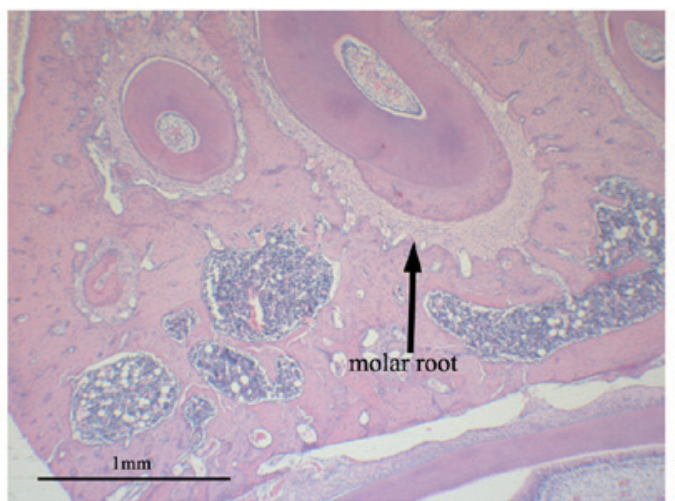

B

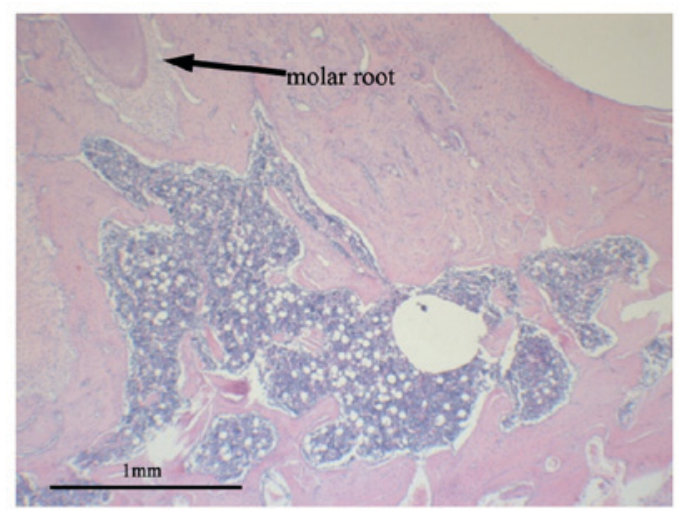

D

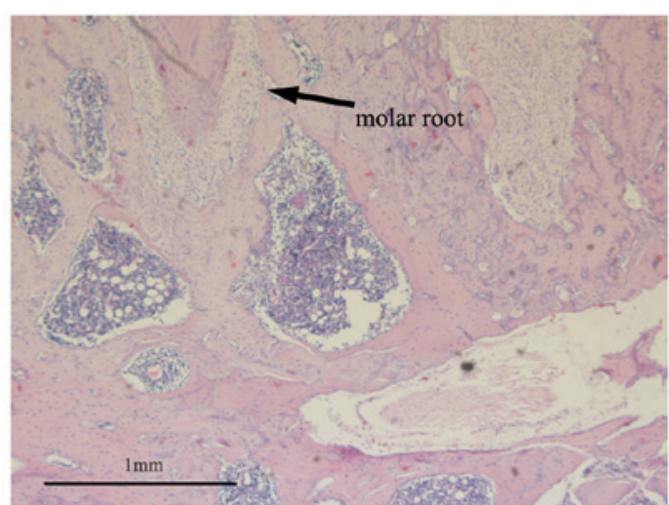

Figure 4. Morphological examination of alveolar bone from the first molar. Hematoxylin and eosin were used for staining of the sections (A) SHAM, (B) OVX, (C) EV and (D) DIO. The Black arrows pointed the first molar roots. Scale bar=1 mm, original magnification, x4. SHAM, sham-operation; DIO, diosgenin; OVX, ovariectomy; EV, estradiol valerate. 
Table I. Key mRNAs associated with the regulatory effect of diosgenin on osteoblasts and osteoclast.

\begin{tabular}{lclc}
\hline Gene Symbol & Fold change & Gene symbol & Fold change \\
\hline Tcf2 & 6.7297165 & Mapk9 & 2.0351413 \\
Alpl & 3.4451298 & Ctnnb1 & 2.0331123 \\
Map2k6 & 3.0244298 & Traf2 & 2.0252916 \\
Smad8 & 2.8872466 & Illb & -14.1060169 \\
Sp7 & 2.8651835 & Tnf & -10.851363 \\
Akt2 & 2.6869522 & Calml3 & -4.2190773 \\
Csnk1al & 2.6036916 & Acp5 & -3.5965811 \\
Ppp3r1 & 2.5592904 & Illr1 & -2.8160289 \\
Spp1 & 2.4752330 & Sost & -2.7645216 \\
Smad4 & 2.4189853 & Fzd9 & -2.3562909 \\
Chuk & 2.3533404 & Tnfrsfla & -2.2219197 \\
Map2k4 & 2.0987597 & Gsk3b & -2.0772502 \\
\end{tabular}

Key $m R N A$ and IncRNA coexpression network associated with regulatory effect of DIO. Considering the positive regulatory effect of DIO on osteoblasts and the negative regulatory effect on osteoclasts shown in the ROOCRA pathway, we selected eight of twenty-four key mRNAs (Ctnnbl, Smad4, Tcf2, Sp7, Illb, Illrl, Tnf and Tnfrsfla) and all of the differentially expressed lncRNAs to construct the coexpression network. A total of 1656 nodes and 5341 edges were obtained in the coexpression network (data not shown).

Pivotal lncRNAs in the coexpression network associated with the regulatory effect of DIO. We regarded six lncRNAs with high closeness scores as the pivotal lncRNAs that could have multiple regulatory effects on mRNAs. The six pivotal IncRNAs are listed in Table II. We further established six modules displaying the interaction between the six pivotal lncRNAs and eight key mRNAs (Fig. 7A-F).

Validation of pivotal lncRNAs and key mRNAs by RT-qPCR. We evaluated the expression of eight key mRNAs and six pivotal lncRNAs in the coexpression network or modules associated with the regulatory effect of DIO. In general, the RT-qPCR data were consistent with the results found in the microarray analysis (Figs. 8 and 9).

\section{Discussion}

Phytoestrogens are a diverse group of plant-derived compounds that can bind to estrogen receptors (ERs) and mimic some actions of estrogen through the activation or inactivation of certain genes (29). It is acknowledged that osteoporosis in postmenopausal women is induced by estrogen deficiency and many phytoestrogens can be used to mitigate osteoporosis. DIO, as a phytoestrogen, had been proven to be a potential anti-osteopenic agent $(30,31)$, but the mechanism of this effect is not fully understood. IncRNAs play a significant role in developing and maintaining the phenotypes or functions of cells (32), including the cells within bone (33). We conducted this study to explore the
Table II. Pivotal lncRNAs in the co-expression network associated with the regulatory effect of diosgenin.

\begin{tabular}{lrl}
\hline lncRNA name & Fold change & Closeness score \\
\hline XR_008346 & -2.1672872 & 788.4166667 \\
MRuc007iji & 2.4346808 & 788.1666667 \\
MRAK157089 & -2.4197937 & 788.1666667 \\
MRAK076413 & 2.5446928 & 788.1666667 \\
MRAK143591 & 2.5193525 & 785.5 \\
AB036696 & -2.2948153 & 780.25 \\
\hline
\end{tabular}

lncRNA, long non-coding RNA.

role of lncRNAs in the anti-osteoporotic influence of DIO in alveolar bone of OVX rats.

Animal models induced by OVX mimics high-turnover bone loss in human such as postmenopausal women. High-turnover bone loss is characterized by excessively high bone formation and bone resorption processes (34). BMD was significantly reduced after ovariectomy as a result of an elevation in alveolar bone turnover among the OVX rats in comparison to the Sham rats. However, the BMD of the alveolar bone was augmented following treatment with EV or DIO in comparison to the OVX group. As plasma biomarkers, levels of both TRAP and ALP were raised in the OVX group rats in comparison to those in the SHAM group. The treatment with EV for 12 weeks lowered the increases in the two biomarkers significantly. It suggested that estrogen was able to lower both bone formation and bone resorption in OVX rats synchronously. Our findings were similar to those of other researchers (35-37). However, DIO not only decreased the level of TRAP but increased the level of ALP (Fig. 1A and B), indicating that DIO only acts as an estrogen-like agent and is not identical to estrogen. DIO may have specific targeting molecules or pathways.

The 3-D bone microstructure analysis using micro-CT demonstrated significant changes in Tb.N, Tb.Th, Tb.Sp, $\mathrm{BV} / \mathrm{TV}$, and SMI, which indicated that there was less loss of alveolar bone in DIO- or EV-treated rats in comparison to the OVX group. The anti-osteoporotic influence of $\mathrm{EV}$ on alveolar bone was more powerful than DIO (Fig. 2). The morphological findings of alveolar bone (Fig. 4) were consistent with the micro-CT evaluation (Fig. 3).

We found that DIO had a strong anti-osteoporotic influence on alveolar bone according to the results from histological observation, micro-CT, the assays of BMD, and bone turnover biomarkers.

We evaluated the IncRNA and mRNA profiles using a microarray in order to confirm the anti-osteoporotic influence of DIO on alveolar bone. As the functions of differentially expressed lncRNAs were largely unclear, we first explored the roles of differentially expressed mRNAs in the anti-osteopenic effects of DIO. The ROOCRA pathway in the IPA database is a unique pathway and covers almost all of the important molecules and signaling associated with functions of osteoblasts and osteoclasts, such as differentiation, mineralization, degradation, development, and apoptosis. 


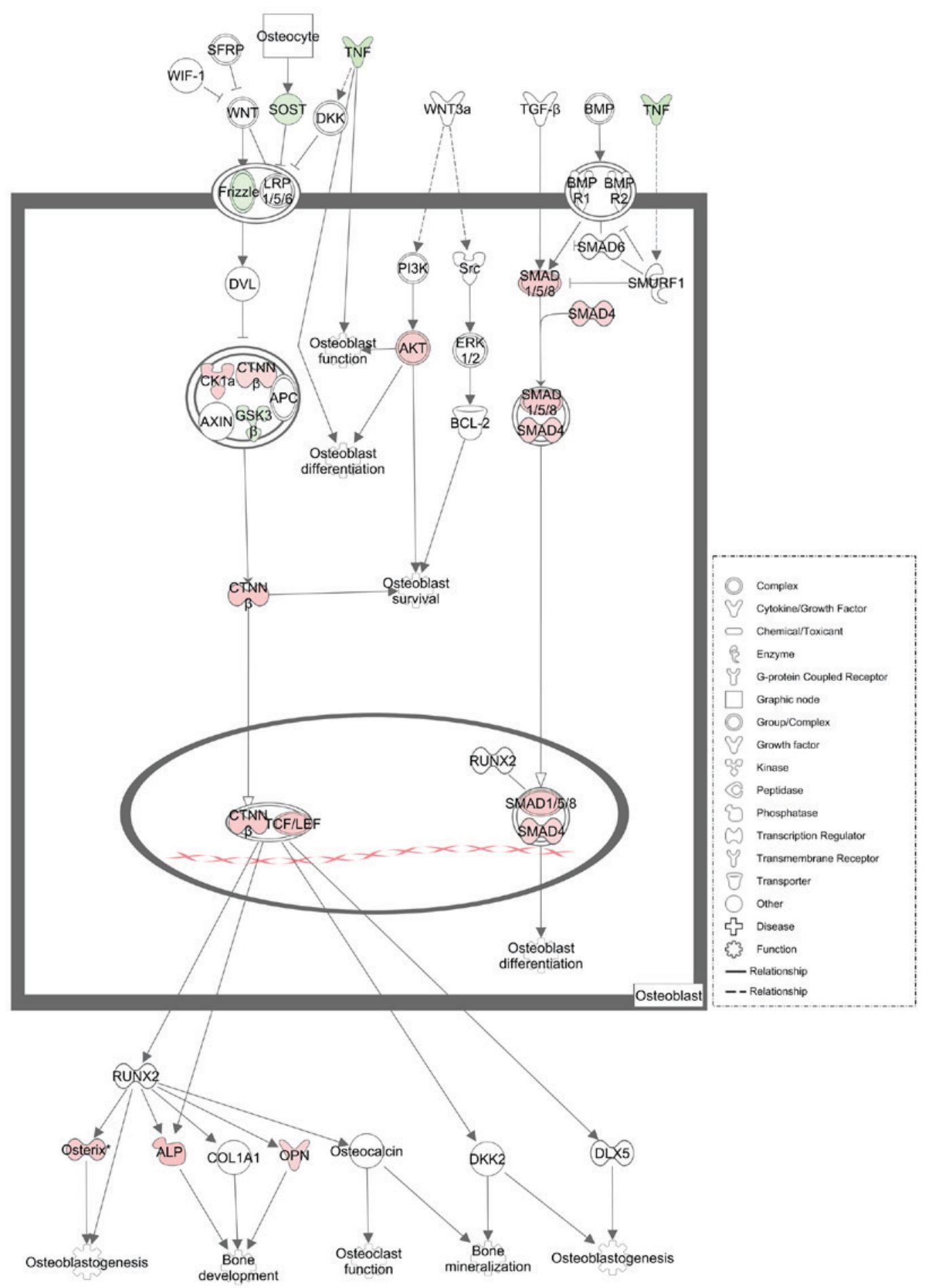

Figure 5. Illustrative diagram elucidating the differentially expressed mRNAs from rat alveolar bone between the DIO group and OVX group in osteoblasts. The genes down-regulated are shown as green, while the genes upregulated are shown as red. White represents genes not chosen but introduced into the network by relation. DIO, diosgenin; OVX, ovariectomy; TNF, tumor necrosis factor; SOST, sclerostin; CK1 $\alpha$, casein kinase 1 alpha; CTNN $\beta$, catenin beta; GSK3 $\beta$, glycogen synthase kinase 3 beta; AKT, serine/threonine kinase; SMAD1/5/8, SMAD family member 1/5/8; SMAD4, SMAD family member 4; TCF/LEF, transcription factor protein; ALP, alkaline phosphatase; OPN, osteopontin.

We found that 24 differentially expressed mRNAs were associated with the ROOCRA pathway (Table I). As shown in Fig. 5, we found that DIO could promote the bone formation process via increasing signaling of the Wnt and BMPs pathways, two recognized signaling pathways regulating the osteogenic differentiation of mesenchymal stem cells or preosteoblasts $(38,39)$. At the mRNA level, the expression of some transcription factors or complexes in osteoblasts, such as Smad4, Smad8, and beta-catenin/Tcf, and the expression of some downstream molecules, such as Osterix, Alp and osteopontin (OPN), were all increased. As shown in Fig. 6, we found that DIO could inhibit the bone resorption process via decreasing two potent stimulators of osteoclastogenesis [tumor necrosis factor-alpha (TNF-alpha) and 


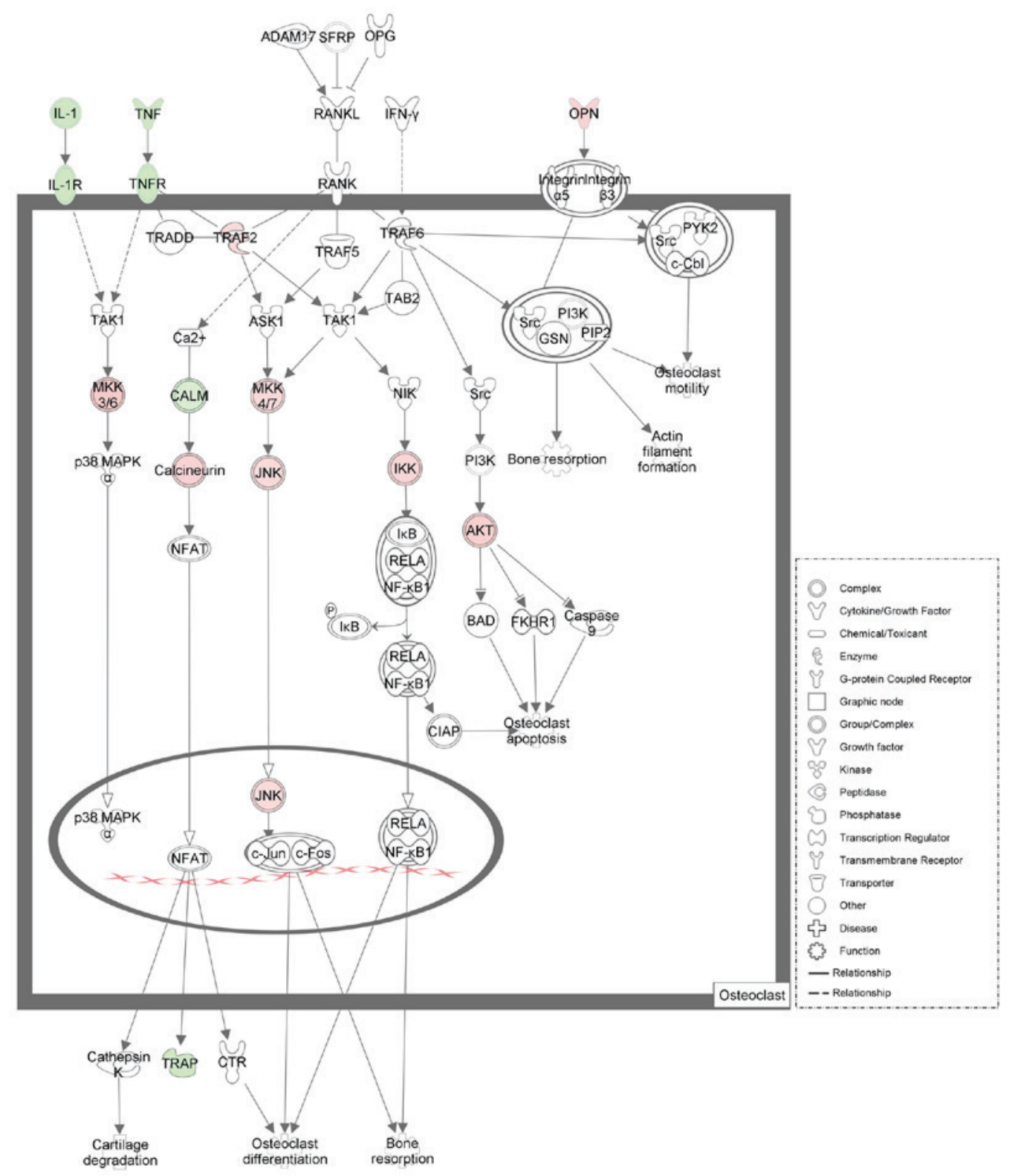

Figure 6. Illustrative diagram elucidating the differentially expressed mRNAs from rat alveolar bone between the DIO group and OVX group in osteoclasts. The genes down-regulated are shown as green, while the genes upregulated are shown as red. White represents genes not chosen but introduced into the network by relation. DIO, diosgenin; OVX, ovariectomy; IL-1, interleukin 1; IL-1R, interleukin 1 receptor; TNF, tumor necrosis factor; TNFR, tumor necrosis factor receptor; OPN, osteopontin; TRAF2, TNF receptor associated factor 2; MKK3/6, mitogen-activated protein kinase kinase 3/6; MKK4/7, mitogen-activated protein kinase kinase 4/7; CALM, calmodulin; JNK, c-Jun N-terminal kinase; IKK, I-kappaB kinase; AKT, serine/threonine kinase; TRAP, tartrate-resistant acid phosphatase.

interleukin-1 beta (IL-1beta)] and their receptors (IL-1R and TNF-R1) (40,41). Particularly, the results from the microarray assay showed the expression of Illb and Tnf (-14.106-and -10.851-fold changes, respectively) were strongly inhibited by DIO. In addition, the mRNA expression of TRAP in alveolar bone was shown to be down-regulated after a 12-week DIO treatment. In brief, the results from the microarray and pathway analysis suggested that the anti-osteoporotic influence of DIO on alveolar bone was attributed to enhanced bone formation via increasing the expression of some transcription factors (e.g., Ctnnb1, Tcf2, Smad4, and Smad8) and to a lowered bone resorption via decreasing the expression of some proinflammatory cytokines or receptors (e.g., Tnf, Illb, Illrl, and Tnfrsfla).
Based on results from the pathway analysis of differentially expressed mRNAs, we focused on eight key mRNAs (Ctnnbl, Tcf2, Smad4, Smad8, Tnf, Illb, Illr1, and Tnfrsfla) and further established a IncRNA-mRNA coexpression network associated with the anti-bone loss effect of DIO to identify pivotal lncRNAs that could regulate the eight key mRNAs mentioned above.

A large coexpression network consisting of 1656 nodes and 5341 edges was constructed. However, almost all of the IncRNAs in the coexpression network were not annotated. This was a limitation of our study. Fortunately, some researchers hold that hub analysis of the coexpression network could overcome this limitation to some degree $(42,43)$. Closeness is one of the most important metrics for finding 
$\mathbf{A}$
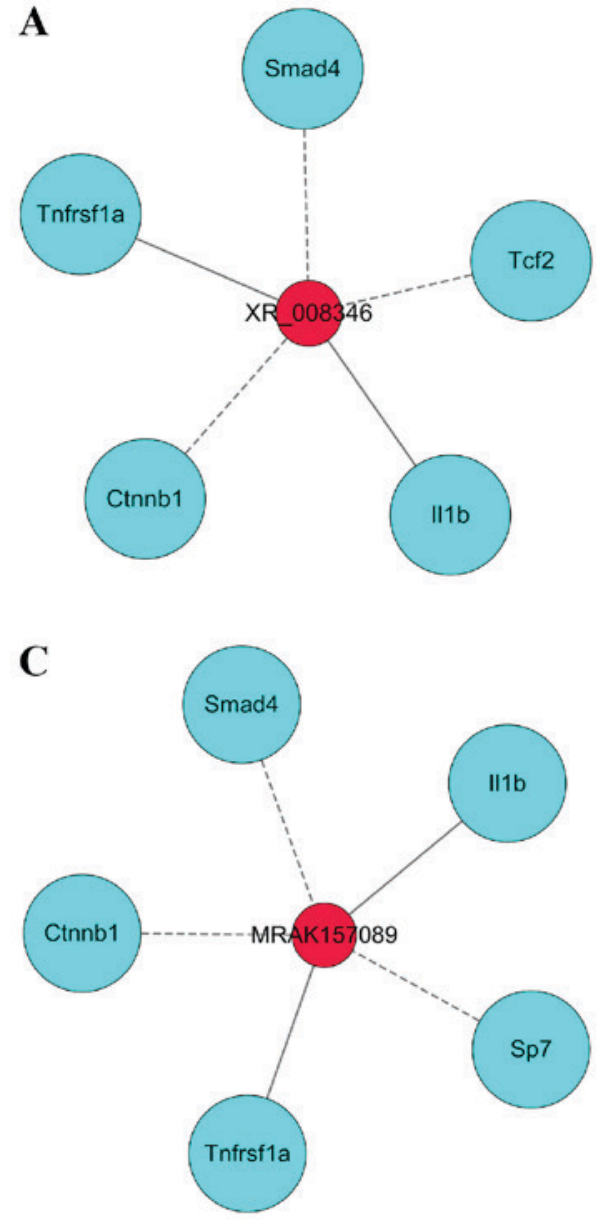

$\mathbf{E}$

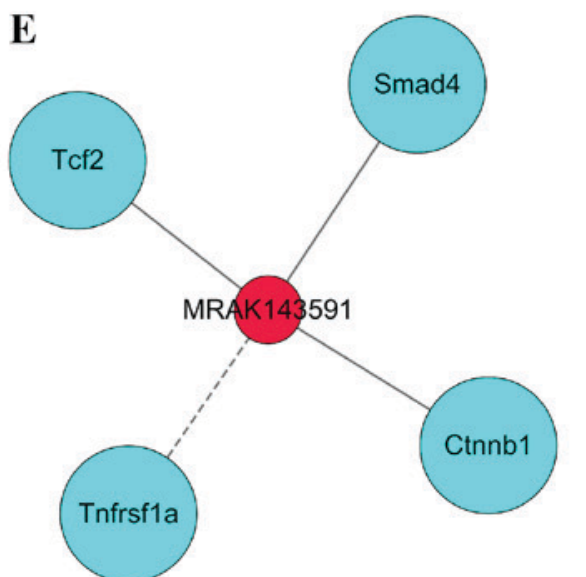

B

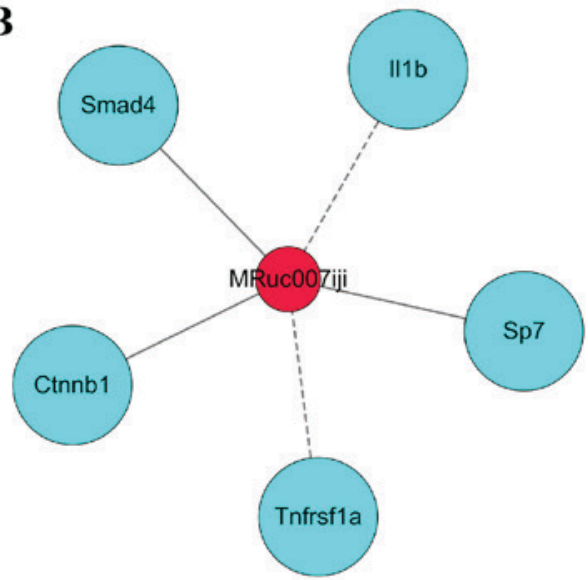

D

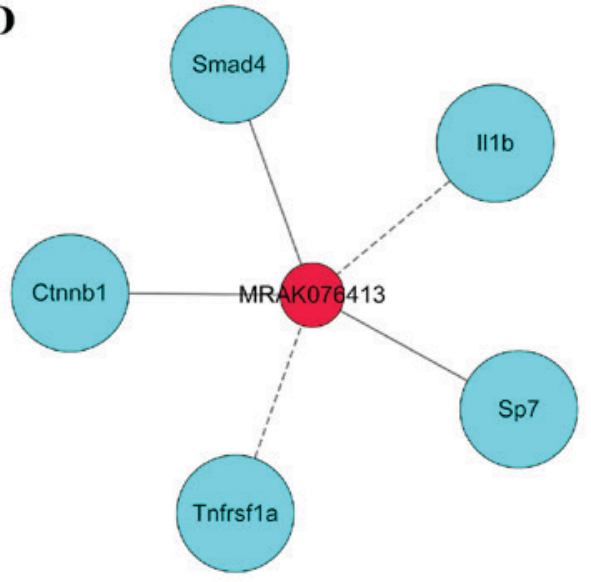

$\mathbf{F}$

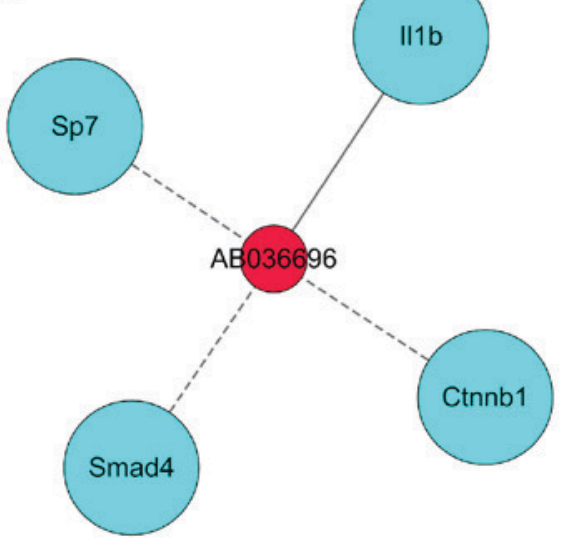

Figure 7. Six modules illustrating interactions between pivotal lncRNAs and key mRNAs in the coexpression network associated with the regulatory effect of DIO. The blue node represents key mRNAs, and the red node represents pivotal lncRNAs. The solid lines indicate a positive correlation, and the dotted lines indicate a negative correlation between lncRNAs and mRNAs. (A) module of XR_008346, (B) module of MRuc007iji, (C) module of MRAK157089, (D) module of MRAK076413, (E) module of MRAK143591 and (F) module of AB036696. DIO, diosgenin.

hubs in a network $(27,44)$. Closeness is the length of the shortest path from one node to another. To evaluate how close any one lncRNA is to eight key mRNAs in this network, we used a closeness score to find the lncRNAs that are most likely to be regulators of the eight key mRNAs. Six pivotal lncRNAs with the highest closeness scores (XR_008346, MRuc007iji, MRAK157089, MRAK076413, MRAK143591, and AB036696) were found. Then, we built six modules that hint at which of these pivotal lncRNAs might have positive or negative regulatory effects on key mRNAs, but we did not find any reports on the relationships between pivotal lncRNAs and key mRNAs. In the future, to reveal the underlying mechanisms of these lncRNAs, further research is necessary.

In conclusion, DIO inhibits ovariectomy-induced loss of alveolar bone in rats via promoting bone formation and inhibiting bone resorption. The mechanism of this anti-osteoporotic influence of DIO probably lies in the global modulation of 

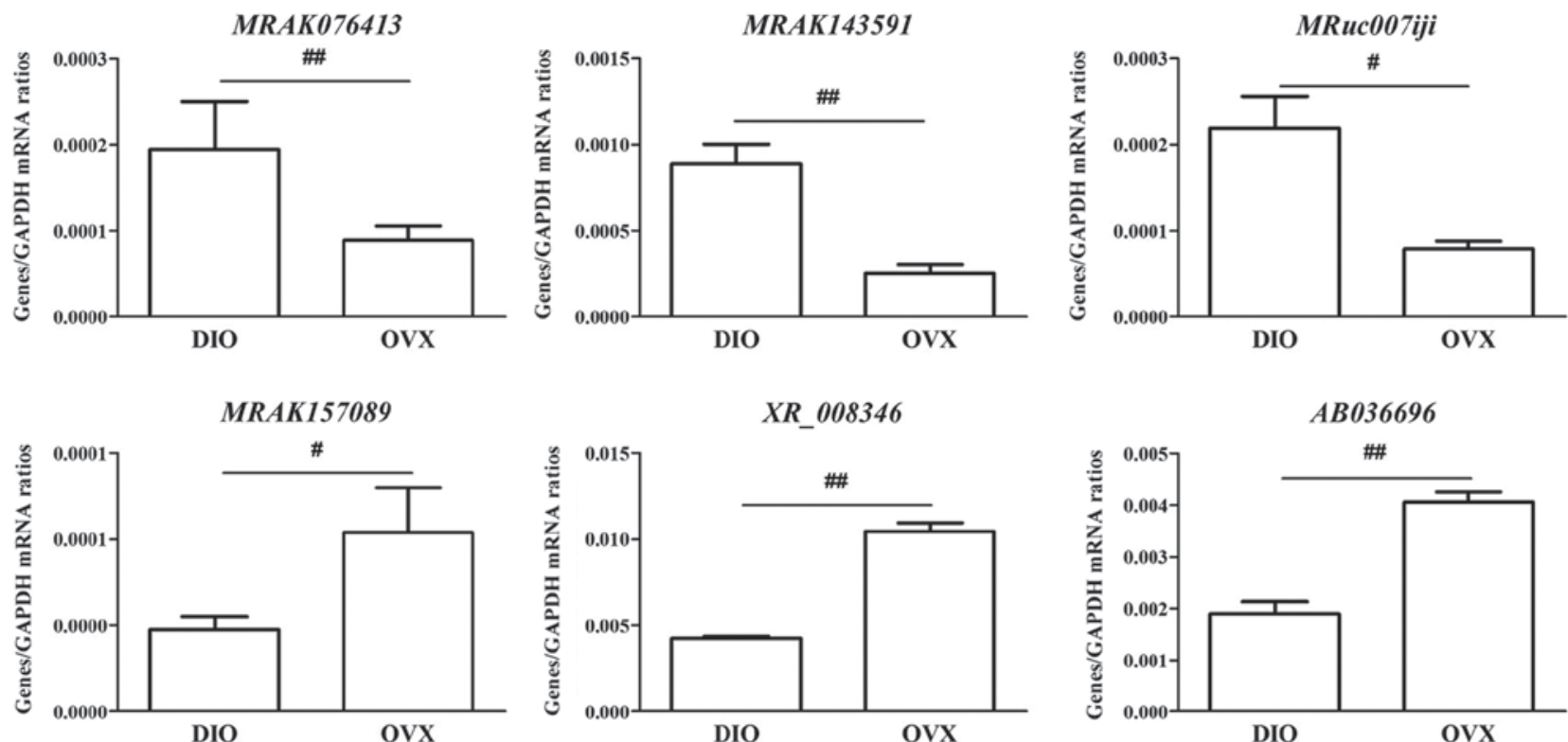

Figure 8. Validation of 6 pivotal lncRNAs using RT-qPCR. Influence of DIO on gene expression of XR_0 08346, MRuc007iji, MRAK157089, MRAK076413, MRAK143591 and AB036696. ${ }^{\# /} \mathrm{P}<0.01$ vs. OVX group; ${ }^{~ P}<0.05$ vs. OVX group. OVX, ovariectomy; DIO, diosgenin.
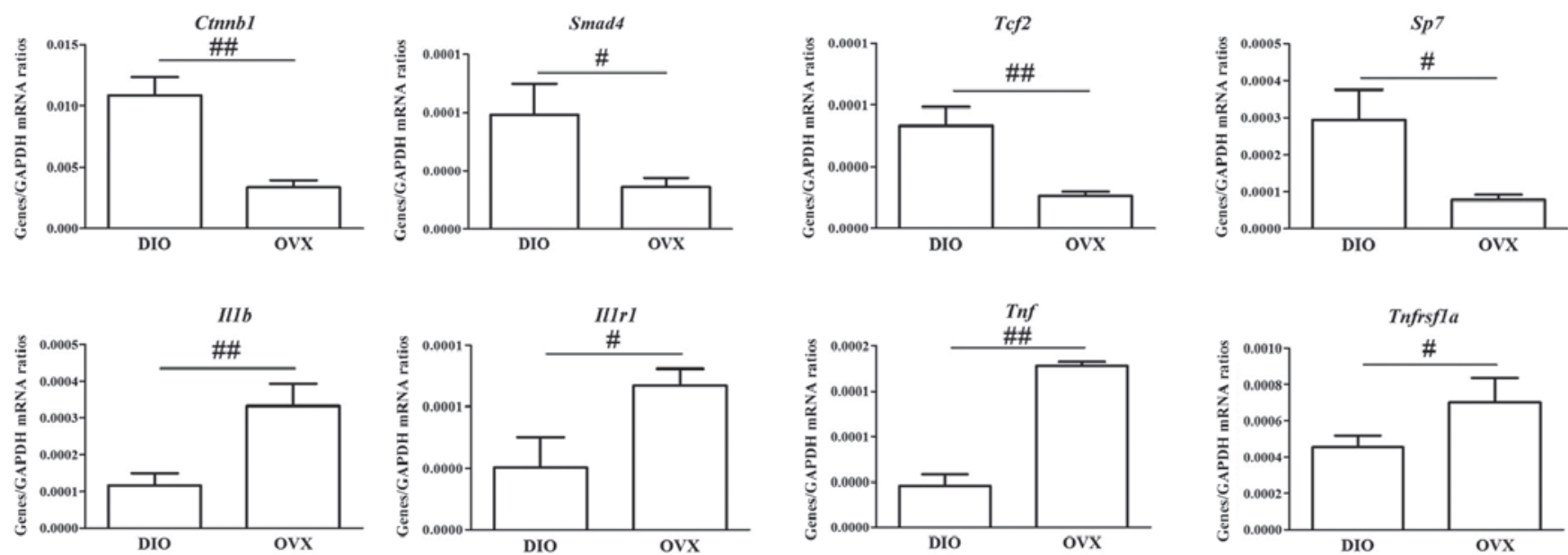

Figure 9. Validation of 8 key mRNAs using RT-qPCR. Influence of DIO on gene expression of Ctnnb1, Smad4, Tcf2, Sp7, Illb, Illr1, Tnf and Tnfrsf1a. "\# $<<0.01$ vs. OVX group; ${ }^{\#} \mathrm{P}<0.05$ vs. OVX group. OVX, ovariectomy; DIO, diosgenin.

the mRNA and IncRNA expression profiles. Of note, six pivotal lncRNAs (XR_008346, MRuc007iji, MRAK157089, MRAK076413, MRAK143591 and AB036696), which may regulate the expression of eight key mRNAs (Ctnnb1, Tcf2, Smad4, Smad8, Tnf, Illb, Illrl and Tnfrsfla), play crucial roles in this process. Our study indicates that DIO can potentially be used as a drug or health supplements for postmenopausal females with alveolar bone loss.

\section{Acknowledgements}

Not applicable.

\section{Funding}

The present study was supported by grant from the National Natural Science Foundation of China (grant no. 81473450), the
Beijing Foundation for Science and Technology Development of Traditional Chinese Medicine (grant no. JJ2015-54) and the Fundamental Research Funds for the Central Public Welfare Research Institutes (grant no. YZ-1780).

\section{Availability of data and materials}

The datasets used and/or analyzed during the current study are available from the corresponding author on reasonable request.

\section{Authors' contributions}

ZZ, GGX and DJ were involved in the study conception and design. ZZ, YC and LX were involved in analysis and interpretation of data. ZZ, YC, GGX and ZW collected data. ZZ, GGX and DJ wrote the article. All authors read and approved the final manuscript. 


\section{Ethics approval and consent to participate}

The animal use protocol has been reviewed and approved by Institutional Ethics Committee of the Institute of Basic Theory, China Academy of Chinese Medical Sciences (Beijing, China).

\section{Patient consent for publication}

Not applicable.

\section{Competing interests}

The authors declare that they have no competing interests.

\section{References}

1. Taguchi A, Tanimoto K, Suei Y and Wada T: Tooth loss and mandibular osteopenia. Oral Surg Oral Med Oral Pathol Oral Radiol Endod 79: 127-132, 1995.

2. Krall EA, Garcia RI and Dawson-Hughes B: Increased risk of tooth loss is related to bone loss at the whole body, hip and spine. Calcif Tissue Int 59: 433-437, 1996.

3. Ikeo T, Goda S and Domae E: Metabolism of alveolar bone. Clin Calcium 16: 117-121, 2006 (In Japanese).

4. Au AL, Kwok CC, Lee AT, Kwan YW, Lee MM, Zhang RZ, Ngai SM, Lee SM, He GW and Fung KP: Activation of iberiotoxin-sensitive, $\mathrm{Ca} 2+$-activated $\mathrm{K}+$ channels of porcine isolated left anterior descending coronary artery by diosgenin. Eur J Pharmacol 502: 123-133, 2004

5. Jesus M, Martins AP, Gallardo E and Silvestre S: Diosgenin: Recent highlights on pharmacology and analytical methodology. J Anal Methods Chem 2016: 4156293, 2016.

6. Medigović I, Ristić N, Živanović J, Šošić-Jurjević B, Filipović B, Milošević V and Nestorović N: Diosgenin does not express estrogenic activity: A uterotrophic assay. Can J Physiol Pharmacol 92: 292-298, 2014.

7. Aradhana, Rao AR and Kale RK: Diosgenin-a growth stimulator of mammary gland of ovariectomized mouse. Indian J Exp Biol 30: 367-370, 1992.

8. Zhang Z, Song C, Fu X, Liu M, Li Y, Pan J, Liu H, Wang S, Xiang L, Xiao GG and Ju D: High-dose diosgenin reduces bone loss in ovariectomized rats via attenuation of the RANKL/OPG ratio. Int J Mol Sci 15: 17130-17147, 2014

9. Folwarczna J, Zych M, Nowinska B, Pytlik M, Bialik M, Jagusiak A, Lipecka-Karcz M and Matysiak M: Effect of diosgenin, a steroidal sapogenin, on the rat skeletal system. Acta Biochim Pol 63: 287-295, 2016.

10. Brown CJ, Ballabio A, Rupert JL, Lafreniere RG, Grompe M, Tonlorenzi R and Willard HF: A gene from the region of the human $\mathrm{X}$ inactivation centre is expressed exclusively from the inactive X chromosome. Nature 349: 38-44, 1991.

11. Bartolomei MS, Zemel S and Tilghman SM: Parental imprinting of the mouse H19 gene. Nature 351: 153-155, 1991.

12. Kwok ZH and Tay Y: Long noncoding RNAs: Lincs between human health and disease. Biochem Soc Trans 45: 805-812, 2017.

13. Deniz E and Erman B: Long noncoding RNA (lincRNA), a new paradigm in gene expression control. Funct Integr Genomics 17: $135-143,2017$.

14. Wei B, Wei W, Zhao B, Guo X and Liu S: Long non-coding RNA HOTAIR inhibits miR-17-5p to regulate osteogenic differentiation and proliferation in non-traumatic osteonecrosis of femoral head. PLoS One 12: e0169097, 2017.

15. Xing D, Liang JQ, Li Y, Lu J, Jia HB, Xu LY and Ma XL: Identification of long noncoding RNA associated with osteoarthritis in humans. Orthop Surg 6: 288-293, 2014.

16. Wang Q, Li Y, Zhang Y, Ma L, Lin L, Meng J, Jiang L, Wang L, Zhou $\mathrm{P}$ and Zhang Y: LncRNA MEG3 inhibited osteogenic differentiation of bone marrow mesenchymal stem cells from postmenopausal osteoporosis by targeting miR-133a-3p. Biomed Pharmacother 89: 1178-1186, 2017

17. Tong $\mathrm{X}, \mathrm{Gu} \mathrm{PC}, \mathrm{Xu} \mathrm{SZ}$ and Lin $\mathrm{XJ}$ : Long non-coding RNA-DANCR in human circulating monocytes: A potential biomarker associated with postmenopausal osteoporosis. Biosci Biotechnol Biochem 79: 732-737, 2015.
18. Hao L, Fu J, Tian Y and Wu J: Systematic analysis of lncRNAs, miRNAs and mRNAs for the identification of biomarkers for osteoporosis in the mandible of ovariectomized mice. Int J Mol Med 40: 689-702, 2017.

19. Li CM, Dong XL, Fan XD, Wu JH, Wang QH, Tian XL, Guo DJ, Wong MS, Qiu TQ and Chan SW: Aqueous extract of danshen (Salvia miltiorrhiza Bunge) protects ovariectomized rats fed with high-fat diet from endothelial dysfunction. Menopause 20: 100-109, 2013.

20. Maïmoun L, Brennan-Speranza TC, Rizzoli R and Ammann P Effects of ovariectomy on the changes in microarchitecture and material level properties in response to hind leg disuse in female rats. Bone 51: 586-591, 2012.

21. Lane NE, Yao W, Kinney JH, Modin G, Balooch M and Wronski TJ: Both hPTH(1-34) and bFGF increase trabecular bone mass in osteopenic rats but they have different effects on trabecular bone architecture. J Bone Miner Res 18: 2105-2115, 2003.

22. Yang J, Pham SM and Crabbe DL: High-resolution Micro-CT evaluation of mid- to long-term effects of estrogen deficiency on rat trabecular bone. Acad Radiol 10: 1153-1158, 2003.

23. Bouxsein ML, Boyd SK, Christiansen BA, Guldberg RE, Jepsen KJ and Müller R: Guidelines for assessment of bone microstructure in rodents using micro-computed tomography. J Bone Miner Res 25: 1468-1486, 2010.

24. Yu G, Yao W, Wang J, Ma X, Xiao W, Li H, Xia D, Yang Y, Deng $\mathrm{K}$, Xiao $\mathrm{H}$, et al: LncRNAs expression signatures of renal clear cell carcinoma revealed by microarray. PLoS One 7: e42377, 2012

25. Liao Q, Liu C, Yuan X, Kang S, Miao R, Xiao H, Zhao G, Luo H, $\mathrm{Bu} \mathrm{D}$, Zhao $\mathrm{H}$, et al: Large-scale prediction of long non-coding RNA functions in a coding-non-coding gene co-expression network. Nucleic Acids Res 39: 3864-3878, 2011.

26. Shannon P, Markiel A, Ozier O, Baliga NS, Wang JT, Ramage D, Amin N, Schwikowski B and Ideker T: Cytoscape: A software environment for integrated models of biomolecular interaction networks. Genome Res 13: 2498-2504, 2003.

27. Zhu J, Li C and Ji W: Identification of genes in ulcerative colitis associated colorectal cancer based on centrality analysis of co-expression network. Neoplasma 62: 756-764, 2015.

28. Chin $\mathrm{CH}$, Chen $\mathrm{SH}, \mathrm{Wu} \mathrm{HH}$, Ho CW, Ko MT and Lin CY: CytoHubba: Identifying hub objects and sub-networks from complex interactome. BMC Syst Biol 8 (Suppl 4): S11, 2014

29. Turner JV, Agatonovic-Kustrin S and Glass BD: Molecular aspects of phytoestrogen selective binding at estrogen receptors. J Pharm Sci 96: 1879-1885, 2007.

30. Hung YT, Tikhonova MA, Ding SJ, Kao PF, Lan HH, Liao JM, Chen JH, Amstislavskaya TG and Ho YJ: Effects of chronic treatment with diosgenin on bone loss in a d-galactose-induced aging rat model. Chin J Physiol 57: 121-127, 2014.

31. Zhao S, Niu F, Xu CY, Liu Y, Ye L, Bi GB, Chen L, Tian G and Nie TH: Diosgenin prevents bone loss on retinoic acid-induced osteoporosis in rats. Ir J Med Sci 185: 581-587, 2016.

32. Sun M and Kraus WL: From discovery to function: the expanding roles of long noncoding RNAs in physiology and disease. Endocr Rev 36: 25-64, 2015.

33. Huynh NP, Anderson BA, Guilak F and McAlinden A: Emerging roles for long noncoding RNAs in skeletal biology and disease. Connect Tissue Res 58: 116-141, 2017.

34. Yeh JK, Chen MM and Aloia JF: Ovariectomy-induced high turnover in cortical bone is dependent on pituitary hormone in rats. Bone 18: 443-450, 1996

35. Dabbaghmanesh MH, Noorafshan A, Talezadeh $P$, Tanideh N, Koohpeyma F, Iraji A, Bakhshayeshkaram M and Montazeri-Najafabady N: Stereological investigation of the effect of Elaeagnus angustifolia fruit hydroalcoholic extract on osteoporosis in ovariectomized rats. Avicenna J Phytomed 7: 261-274, 2017.

36. Li S, Zhang W, Duan F, Liu W, Sun X and Pan X: The preventive and therapeutic roles of phytoestrogen $\alpha$-Zearalanol on osteoporetic rats due to ovariectomization. Iran J Basic Med Sci 19: 1216-1221, 2016

37. You MK, Kim DW, Jeong KS, Bang MA, Kim HS, Rhuy J and Kim HA: St. John's Wort (Hypericum perforatum) stimulates human osteoblastic MG-63 cell proliferation and attenuates trabecular bone loss induced by ovariectomy. Nutr Res Pract 9: 459-465, 2015.

38. Marcellini S, Henriquez JP and Bertin A: Control of osteogenesis by the canonical Wnt and BMP pathways in vivo: Cooperation and antagonism between the canonical Wnt and BMP pathways as cells differentiate from osteochondroprogenitors to osteoblasts and osteocytes. Bioessays 34: 953-962, 2012. 
39. Lin GL and Hankenson KD: Integration of BMP, Wnt, and notch signaling pathways in osteoblast differentiation. J Cell Biochem 112: 3491-3501, 2011.

40. Kudo O, Fujikawa Y, Itonaga I, Sabokbar A, Torisu T and Athanasou NA: Proinflammatory cytokine (TNFalpha/IL-1alpha) induction of human osteoclast formation. J Pathol 198: 220-227, 2002.

41. Kwan Tat S, Padrines M, Théoleyre S, Heymann D and Fortun Y: IL-6, RANKL, TNF-alpha/IL-1: Interrelations in bone resorption pathophysiology. Cytokine Growth Factor Rev 15: 49-60, 2004.

42. Dou C, Cao Z, Yang B, Ding N, Hou T, Luo F, Kang F, Li J, Yang X, Jiang H, et al: Changing expression profiles of lncRNAs, mRNAs, circRNAs and miRNAs during osteoclastogenesis. Sci Rep 6: 21499, 2016.
43. Wu L, Xu Q, Zhang H, Li M, Zhu C, Jiang M, Sang X, Zhao Y, Sun $Q$ and Zhao H: A new avenue for obtaining insight into the functional characteristics of long noncoding RNAs associated with estrogen receptor signaling. Sci Rep 6: 31716, 2016.

44. Ghosh R and Lerman K: Parameterized centrality metric for network analysis. Phys Rev E Stat Nonlin Soft Matter Phys 83: 066118, 2011.

(i) $($ ) This work is licensed under a Creative Commons Attribution-NonCommercial-NoDerivatives 4.0 International (CC BY-NC-ND 4.0) License. 